\title{
Near-Field-Induced Femtosecond Breakdown of Plasmonic Nanoparticles
}

\author{
Benedek J. Nagy ${ }^{1} \cdot$ Zsuzsanna Pápa $^{1,2} \cdot$ László Péter $^{1} \cdot$ Christine Prietl $^{3} \cdot$ Joachim R. Krenn ${ }^{3} \cdot$ Péter Dombi ${ }^{1,2}$
}

Received: 12 July 2019 / Accepted: 30 September 2019 / Published online: 31 October 2019

(C) The Author(s) 2019

\begin{abstract}
We studied the evolution of femtosecond breakdown in lithographically produced plasmonic nanoparticles with increasing laser intensity. Localized plasmons were generated with 40 -fs laser pulses with up to $1.4 \times 10^{12} \mathrm{~W} / \mathrm{cm}^{2}$ peak intensity. The damage morphology shows substantial variation with intensity, starting with the detachment of hot spots and stochastic nanoparticle removal. For higher intensities, we observe precise nanolithographic mapping of near-field distributions via ablation. The common feature of these phenomena is the central role played by the single plasmonic hot spot of the triangular nanoparticles used. We also derive a damage threshold value from stochastic damage trends on the arrays fostering the optimization of novel nanoarchitectures for nonlinear plasmonics.
\end{abstract}

Keywords Plasmonics $\cdot$ Laser-induced damage threshold $\cdot$ Plasmonic nanoparticles $\cdot$ Field enhancement mapping

Metal nanostructures are central to a wide range of ultrafast plasmonic effects, where the exploitation of nonlinearities require high laser intensities, typically induced by femtosecond laser pulses. Large field enhancement factors can be achieved with the generation of surface plasmons on properly designed nanosystems. Both localized plasmon oscillations and propagating surface plasmon waves are ideal for this purpose $[1,2]$. Plasmonic field enhancement combined with femtosecond laser illumination of samples can be used in many applications, for example, the conversion of the frequency of light to loworder harmonics, $\mathrm{THz}$ radiation, or even high-order harmonic generation [3-12]. In these cases, bow-tie- [5-7], rod- [8], and triangle-shaped [9] nanoparticles are used among others [10-12]. In addition, applications of ultrafast, nonlinear plasmonics involve the construction of nonlinear nanoemitters of electrons [13-16], photoelectron spectroscopy for subnanometer field probing [17], carrier-envelope phase detection [18], and many more [19-22]. In all of these cases, the high field enhancement combined with the usage of

Benedek J. Nagy

nagy.benedek@wigner.mta.hu

Wigner Research Centre for Physics, Budapest, Hungary

ELI-ALPS Research Institute, Szeged, Hungary

3 Institut für Physik, Karl-Franzens-Universität, Graz, Austria femtosecond pulses can lead to the rapid breakdown of plasmonic structures.

In order to exploit plasmonic nonlinearities as much as possible (i.e., to maximize harmonic signal yield, photocurrent, etc.), a detailed understanding of the laser-induced breakdown of the nanostructures is necessary. Therefore, it is essential to know the accurate laser-induced damage threshold (LIDT) for lithographically produced plasmonic samples. Staying below the LIDT during the measurements can increase the lifetime of the samples and help maintain their stability in application environments.

In contrast to typical femtosecond damage tests on laser mirrors [23-25], real-time plasmonic damage monitoring is hindered by the fact that nanostructures are too small for direct observation with optical methods (such as optical microscopy), and many of the applications involve a vacuum environment which renders sample state monitoring even more difficult. Despite the importance of the plasmonic damage problem, there are only few systematic studies of the damage behavior of such nanoparticles. Femtosecond laser-induced damage on nanostructures was observed in the case of high harmonic generation where the nanorod edges became smoother after exposure [3] or nanoparticles are damaged [26] or simply melt [27].

Previously, damage on plasmonic nanorods was quantified by counting based on the average number of nanoparticles removed from the substrate [28] and nanoholes were fabricated well above the damage threshold [29]. Laser-induced reshaping of nanoparticles does not properly indicate damage 


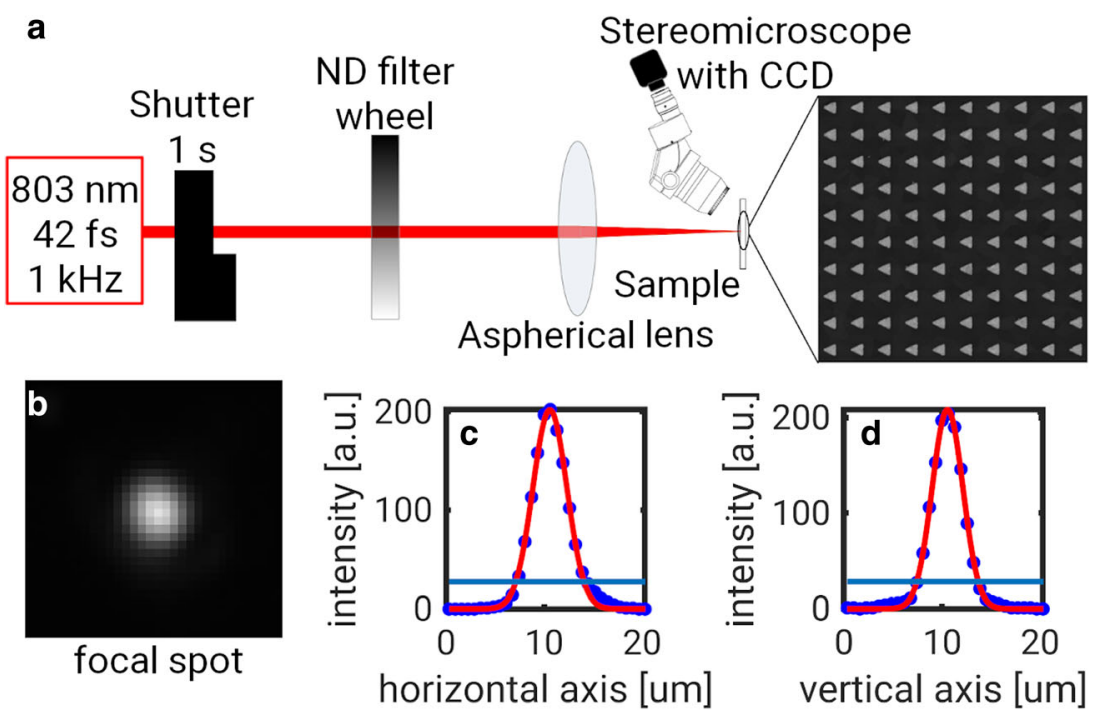

Fig. 1 a Scheme of the experimental setup: a regenerative Ti:sapphire amplifier with $803-\mathrm{nm}$ central wavelength, a shutter set to $1 \mathrm{~s}$ opening time, and a continuously variable neutral density (ND) filter wheel. The sample was mounted on a $3 \mathrm{D}$ piezo translation stage to control the sample position, and a microscope equipped with a CCD was used for damage monitoring. The SEM image shows the nanoparticle array. b Focal spot on a CCD, $\mathbf{c}-\mathbf{d}$ Gaussian fit (red) onto the linecuts (blue symbols) of the beam profile in $\mathbf{c}$ vertical and $\mathbf{d}$ horizontal directions. The blue lines correspond to the $1 / e^{2}$ level of the Gaussian peak

power levels were set by a variable neutral density filter. We applied normal incidence and the sample was mounted on an xyz piezo translation stage. A microscope equipped with a CCD camera was used to determine the laser beam focus position on the sample.

The dimensions of the nanoparticles used in our test setup are shown in Fig. 2a. Each array has $0.01-\mathrm{mm}^{2}$ surface area with the particles arranged in a quadratic grid of $350-\mathrm{nm}$ pitch in both directions. The nanoparticles are deposited on a fused silica substrate with a conductive indium-tin-oxide (ITO) thin film on top. The achieved extinction spectrum is shown in Fig. $2 b$ together with the spectrum of the excitation laser pulses.

The damage measurement procedure consisted of moving the sample with the positioning stage laterally and irradiating different parts of the nanoparticle array with 1000 shots for each laser intensity value. Subsequently, we moved the sample to a new (nonirradiated) spot and increased the intensity. We repeated the procedure after investigating the damage sites with a scanning electron microscope (SEM) and readjusted the used intensity levels according to SEM images of the damaged sites. The criteria for these readjustments were
Fig. 2 a Dimensions of the plasmonic nanoparticle and laser polarization direction (red arrow). b Extinction spectrum of the plasmonic nanoparticle (purple) and the laser spectrum (red)
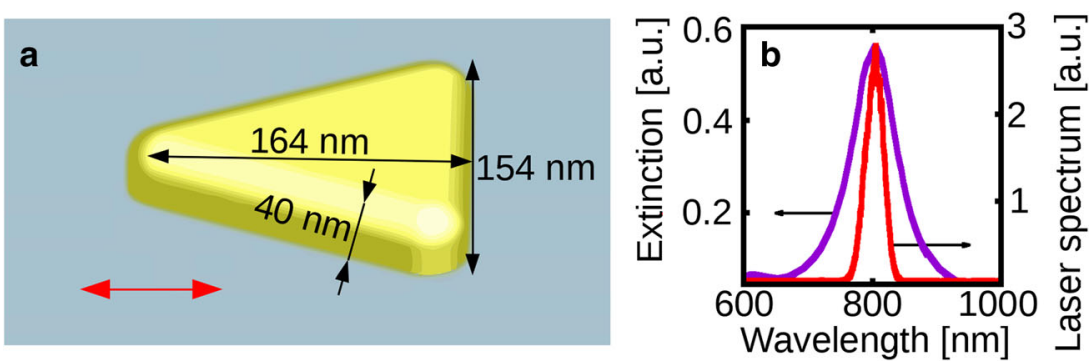


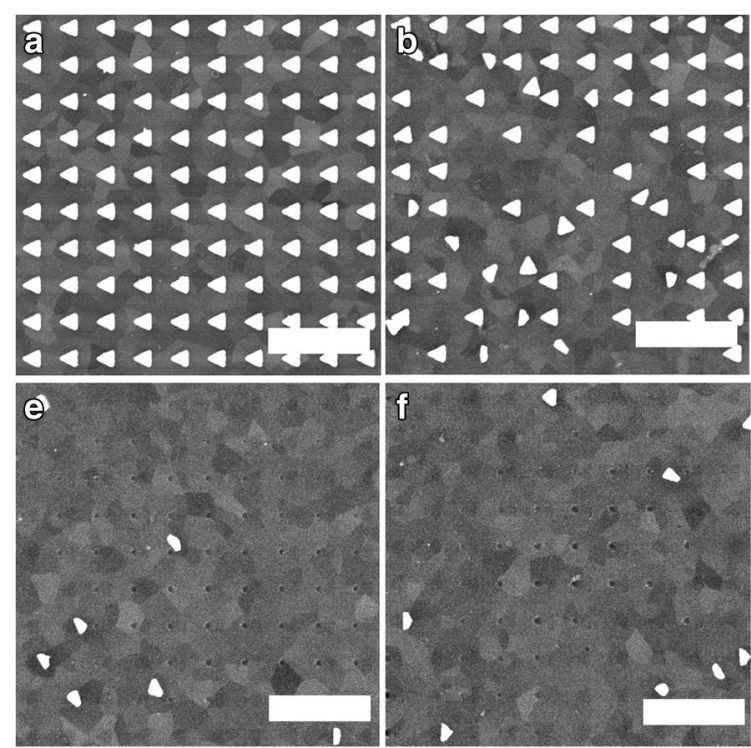

Fig. 3 SEM images of samples irradiated with different laser fluences. The scale bar is $1 \mu \mathrm{m}$, which also indicates the polarization direction of the laser beam. a The central area of the laser focal spot on an intact sample illuminated with $2.0 \mathrm{~mJ} / \mathrm{cm}^{2}$. b Some nanoparticles relocated at $3.3 \mathrm{~mJ} / \mathrm{cm}^{2}$. c More nanoparticles detached at $6.5 \mathrm{~mJ} / \mathrm{cm}^{2}$. d Small damage spots start to appear at the apex's original position on the

whether in the applied intensity range, the outcome covered all of the scenarios shown in Fig. 3. After a few readjustments, we were able to determine an intensity range at which we performed several new measurements. The resulting intensity scan can be seen in Fig. 3. After having performed the final femtosecond damage test, SEM images were recorded with a TESCAN MIRA3 instrument. An acceleration voltage of $18 \mathrm{kV}$ proved to be ideal for obtaining images with the highest sharpness. For enhancing the composition contrast, mixed backscattered/secondary images were obtained with an 80 / 20 blending ratio.

With the laser peak fluence ranging from 2.0 to $114.3 \mathrm{~mJ} /$ $\mathrm{cm}^{2}$, we recorded damage phenomena for 8 different

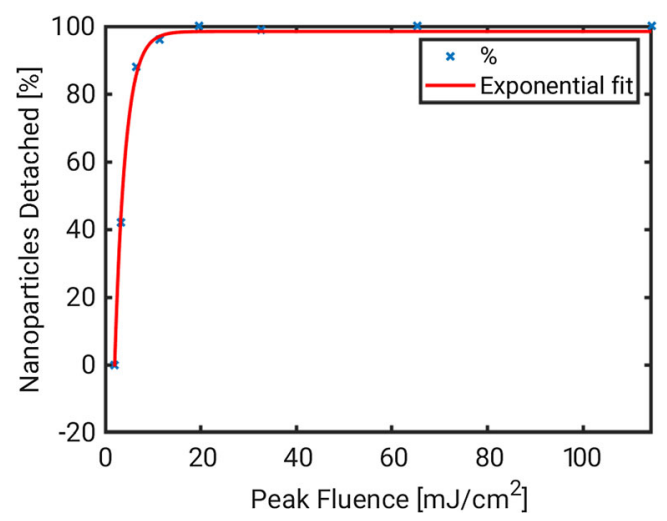

Fig. 4 Percentage of stochastically detached nanoparticles. An exponential curve was fitted to the data which intersects the $0 \%$ damage threshold line at $2.0 \pm 0.4 \mathrm{~mJ} / \mathrm{cm}^{2}$. This way, we define as the local fieldinduced damage threshold for plasmonic nanoparticles

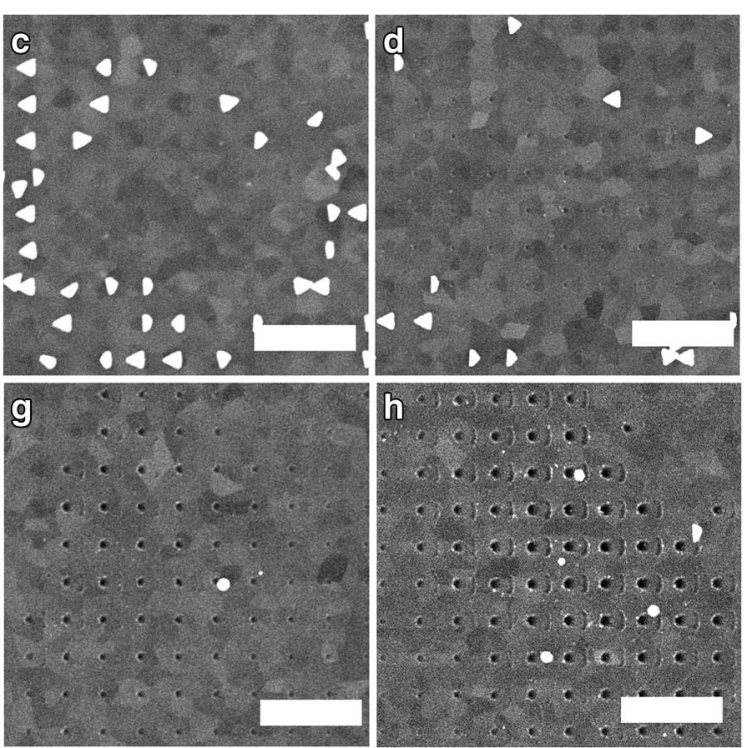

substrate at $11.4 \mathrm{~mJ} / \mathrm{cm}^{2}$. e Larger spots on the substrate at $19.6 \mathrm{~mJ} /$ $\mathrm{cm}^{2}$. f Damage spots also show occasionally the base ends of the triangles at $32.7 \mathrm{~mJ} / \mathrm{cm}^{2}$. g Base end spots are shown more often at $65.3 \mathrm{~mJ} / \mathrm{cm}^{2}$. h Also, the contour of the triangles is visible at $114.3 \mathrm{~mJ} /$ $\mathrm{cm}^{2}$, the damage spot is at the hot spot of the triangle, and the base is also visible due to the field enhancement on the base ends

intensities, shown in Fig. 3. At the lowest peak fluence, the sample remained intact (Fig. 3a). By increasing the laser intensity, some particles became detached and relocated (Fig. $3 \mathrm{~b}, \mathrm{c})$. At $11.4 \mathrm{~mJ} / \mathrm{cm}^{2}$ small damaged spots emerged on the substrate (Fig 3d) with their size increasing for higher intensities (Fig 3e). For the highest intensities, the whole contour of the triangles starts to appear as a lithographically produced shape in the surface of the substrate (Fig. $3 \mathrm{f}-\mathrm{h}$ ).

We can determine a damage threshold in two ways. First, we can take into account that the smallest peak fluence applied was $2.0 \mathrm{~mJ} / \mathrm{cm}^{2}$ where no damage occurred (Fig. 3a). The next fluence step that we tested was $3.3 \mathrm{~mJ} / \mathrm{cm}^{2}$ where damage is clearly observed in the SEM images (Fig. 3b). Hence, the approximate damage threshold can be calculated as the average of these peak fluences yielding a LIDT of $2.75 \pm 0.70 \mathrm{~mJ} /$ $\mathrm{cm}^{2}$. Another suitable method can be calculating the fluencedependent ratio of the detached nanoparticles (more precisely, relocated or substantially distorted/melted nanoparticles) [28]. We apply an exponential fit to the data points and calculate the intersection with the $x$-axis. This way, we arrive at a damage threshold value of $2.0 \pm 0.4 \mathrm{~mJ} / \mathrm{cm}^{2}$ (Fig. 4). These two values are very close to each other, inside within their respective error limits. It is safe to determine the damage threshold as the lower peak fluence of the two. The $2.0 \mathrm{~mJ} / \mathrm{cm}^{2}$ value is comparable with the value $1.2 \mathrm{~mJ} / \mathrm{cm}^{2}$, measured for plasmonic nanorods, with the difference possibly explained by different fabrication conditions and near-field distributions [28].

As we increase the peak fluence, we also see a difference not only in the amount of nanoparticles removed, but also in 
Fig. 5 a SEM image of the contour of the triangle after the exposure with $114.3 \mathrm{~mJ} / \mathrm{cm}^{2}$ (scale bar $50 \mathrm{~nm}$ ). b Finitedifference time-domain simulated electric field enhancement (colorcoded) for the triangular nanoparticle
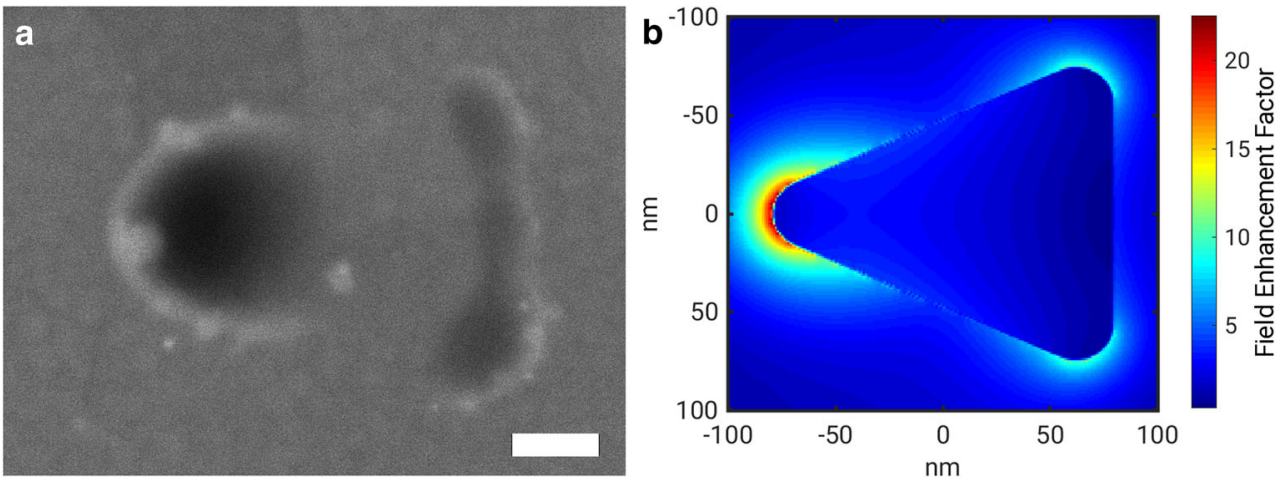

the traces the removed nanoparticles leave behind, as shown in detail in Fig. 3. It is clearly visible that at the highest peak fluence, there are areas where holes appear on the surface of the substrate. It is also clearly visible that damage sets in at the plasmonic hot spot, i.e., at the tip of the triangle first. With increasing fluence, the contour of the whole triangles appears corresponding to near-field field enhancement mapping (Fig. 5a). Further evidence of this is provided by finite-difference time-domain (FDTD) simulations of the near-field distribution in Fig. $5 b$ showing a strikingly good correspondence to the experimentally observed pattern in Fig. 5a. For these simulations, we applied the Lumerical FDTD Solutions software. The particle geometry was determined according to the SEM images. The simulation volume containing one triangle had lateral dimensions of $350 \mathrm{~nm} \times 350 \mathrm{~nm}$ with periodic boundary conditions for mimicking the response of the nanoparticle array. For illumination, we applied a short pulse with the same parameters as our laser source. With the support of the deduced near-field distributions, our experiments thus provide another interesting example of mapping plasmonic near-fields with the help of femtosecond lasers [34]. It is also obvious that the nanoablation process is achieved while nanoparticles are still at their place, as not only the hole at the tip but also the edges can be seen.

We assume that the damage on the substrate is caused by the Joule heat at the tip of the sample during its interaction with the laser pulses. As the triangles are designed to be resonant for the laser wavelength (Fig. 2a), the heating effects can be influenced by matching the resonance with the laser spectrum and lead to a different result regarding heat accumulation as in ref. [35]. We can calculate the near-field enhancement of the triangles for particularly the spectrum of the laser used in the experiment (see Fig. 5b), and this shows a high field enhancement at the tip of the triangle and lower field enhancement generated on the opposite side. By irradiating the nanoparticles with the laser beam, photoelectrons are emitted from the sample [22, 36-38]. The hot spots with the highest enhancement lead to high local current as the triangles recover the charge caused by the leaving photoelectrons through the bias. This high and highly localized current can be responsible for leaving such a mark on the substrate before the triangles become detached from the surface. This current can be also responsible for altering the adhesion properties locally, which leads to the removal of the nanoparticles.

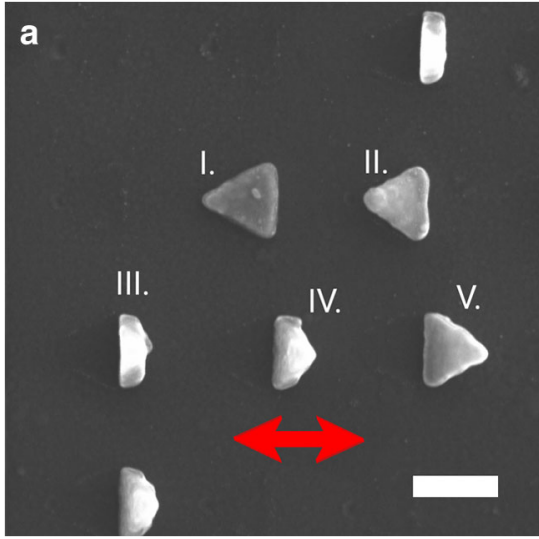

Fig. 6 a, b Two typical images of the nanotriangle array after irradiation with nanoparticle hot spot detachment (scale bar $200 \mathrm{~nm}$ ). The laser polarization is indicated by red arrows. Phases of detachment are identified as follows: (I) nanoparticle remained intact; (II) visible

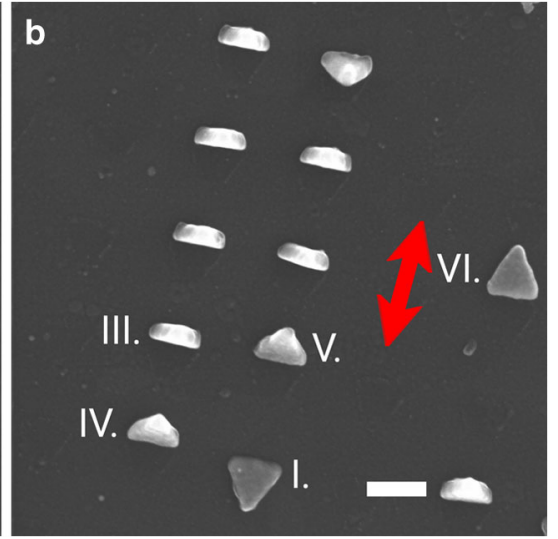

detachment of the hot spot; (III) triangle standing vertical to substrate; (IV, V) triangles flipped over their base; (VI) triangle completely flipped, laying on the substrate again 
Different stages of the detachment within one SEM image are shown in Fig. 6.

In summary, we determined the damage threshold of lithographically produced gold plasmonic nanotriangles as 2.0 $\mathrm{mJ} / \mathrm{cm}^{2}$. For higher intensities, we observed substantially different damage morphologies including nanoparticle flipping, full detachment, hot spot nanoablation, and full-contour ablation (in the order of increasing intensity). With this, we also demonstrated the near-field-induced nature of these damage processes. These results also help the design of more damage resistant samples for ultrafast nonlinear plasmonics applications, the development of nanostructured photocathodes, nearfield-enhanced high harmonic generation, and many more. Since the signal in these applications depends nonlinearly on the laser intensity, a small improvement in the damage resistance of the samples will lead to a significant increase of the intensity of the useful optical or electron signal.

Funding Information Open access funding provided by MTA Wigner Research Centre for Physics (MTA Wigner FK, MTA EK). The authors received funding from the National Research, Development and Innovation Office of Hungary (grants VEKOP-2.3.2-16-2017-00015, 2018-1.2.1-NKP-2018-00012 and 128077), and the PETACom project no. 829153 financed by the FET Open H2020 program.

Open Access This article is distributed under the terms of the Creative Commons Attribution 4.0 International License (http:// creativecommons.org/licenses/by/4.0/), which permits unrestricted use, distribution, and reproduction in any medium, provided you give appropriate credit to the original author(s) and the source, provide a link to the Creative Commons license, and indicate if changes were made.

\section{References}

1. Atwater HA (2007) The promise of plasmonics. Sci. Am. 296:56

2. Schuller JA, Barnard ES, Cai W, Jun YC, White JS, Brongersma ML (2010) Plasmonics for extreme light concentration and manipulation. Nat. Mater. 9:193

3. Vampa G, Ghamsari BG, Mousavi SS, Hammond TJ, Olivieri A, Lisicka-Skrek E, Naumov AY, Villeneuve DM, Staudte A, Berini P, Corkum PB (2017) Plasmon-enhanced high-harmonic generation from silicon. Nat. Phys. 13:659

4. Celebrano $\mathrm{M}$ et al (2015) Mode matching in multiresonant plasmonic nanoantennas for enhanced second harmonic generation. Nat. Nanotechnol. 10:421

5. Hanke T, Krauss G, Träutlein D, Wild B, Bratschitsch R, Leitenstorfer A (2009) Efficient nonlinear light emission of single gold optical antennas driven by few-cycle near-infrared pulses. Phys. Rev. Lett. 103:257404

6. Kim S, Jin J, Kim Y-J, Park I-Y, Kim Y, Kim S-W (2008) Highharmonic generation by resonant plasmon field enhancement. Nature 453:757

7. Sivis M, Duwe M, Abel B, Ropers C (2012) Nanostructureenhanced atomic line emission. Nature 485:E1

8. Metzger B, Hentschel M, Giessen H (2017) Probing the near-field of second-harmonic light around plasmonic nanoantennas. Nano Lett. 17:1931
9. Polyushkin DK, Márton I, Rácz P, Dombi P, Hendry E, Barnes WL (2014) Mechanisms of THz generation from silver nanoparticle and nanohole arrays illuminated by $100 \mathrm{fs}$ pulses of infrared light. Phys. Rev. B 89:125426

10. Hanke T, Cesar J, Knittel V, Trügler A, Hohenester U, Leitenstorfer A, Bratschitsch R (2012) Tailoring spatiotemporal light confinement in single plasmonic nanoantennas. Nano Lett. 12:992-996

11. Sivis M, Ropers C (2013) Generation and bistability of a waveguide nanoplasma observed by enhanced extreme-ultraviolet fluorescence. Phys. Rev. Lett. 111:085001

12. Iwaszczuk K, Zalkovskij M, Strikwerda AC, Jepsen PU (2015) Nitrogen plasma formation through terahertz-induced ultrafast electron field emission. Optica 2:116

13. Tsujino S, Beaud P, Kirk E, Vogel T, Sehr H, Gobrecht J, Wrulich A (2008) Ultrafast electron emission from metallic nanotip arrays induced by near infrared femtosecond laser pulses. Appl. Phys. Lett. 92:193501

14. Li RK, H. To, Andonian G, Feng J, Polyakov A, Scoby CM, Thompson K, Wan W, Padmore HA, Musumeci P (2013) Surface-plasmon resonance-enhanced multiphoton emission of high-brightness electron beams from a nanostructured copper cathode. Phys. Rev. Lett. 110:074801

15. Vogelsang J, Robin J, Nagy BJ, Dombi P, Rosenkranz D, Schiek M, Groß P, Lienau C (2015) Ultrafast electron emission from a sharp metal nanotaper driven by adiabatic nanofocusing of surface plasmons. Nano Lett. 15:4685-4691

16. Hobbs RG, Yang Y, Fallahi A, Keathley PD, De Leo E, Kärtner FX, Graves WS, Berggren KK (2014) High-yield, ultrafast, surface plasmon-enhanced, Au nanorod optical field electron emitter arrays. ACS Nano 8:11474-11482

17. Rácz P, Pápa Z, Márton I, Budai J, Wróbel P, Stefaniuk T, Prietl C, Krenn JR, Dombi P (2017) Measurement of nanoplasmonic field enhancement with ultrafast photoemission. Nano Lett 17(2):1181-1186

18. Putnam WP, Hobbs RG, Keathley PD, Berggren KK, Kärtner FX (2017) Optical-field-controlled photoemission from plasmonic nanoparticles. Nat. Phys 13(4):335

19. Stockman MI (2011) Nanoplasmonics: past, present, and glimpse into future. Opt. Express 19:22029

20. Ahn B, Schötz J, Okell WA, Süßmann F, Förg B, Kim SC, Kling MF, Kim D (2016) Optimization of a nanotip on a surface for the ultrafast probing of propagating surface plasmons. Opt. Express 24:92

21. Ahn B et al (2017) Attosecond-controlled photoemission from metal nanowire tips in the few-electron regime. APL Photonics 2: 036104

22. Dombi P, Hörl A, Rácz P, Márton I, Trügler A, Krenn JR, Hohenester U (2013) Ultrafast strong-field photoemission from plasmonic nanoparticles. Nano Lett. 13:674

23. B. J. Nagy, L. Vámos, D. Oszetzky, P. Rácz, L. Gallais, P. Dombi, "Direct comparison of kilohertz- and megahertz-repetition-rate femtosecond damage threshold", Opt. Lett. 40, 2525-2528, (2015) and references therein.

24. Csajbók V, Szikszai L, Nagy BJ, Dombi P (2016) Femtosecond damage resistance of femtosecond multilayer and hybrid mirrors. Opt. Lett 41:3527-3530

25. Csajbók V, Bedőházi Z, Nagy BJ, Dombi P (2018) Ultrafast multipulse damage threshold of femtosecond high reflectors. Appl. Optics 57:340-343

26. Liu $\mathrm{H}$ et al (2018) Enhanced high-harmonic generation from an alldielectric metasurface. Nat. Phys. 14:1006-1010

27. Pfullmann $\mathrm{N}$ et al (2013) Bow-tie nano-antenna assisted generation of extreme ultraviolet radiation. New J. Phys. 15:1367-2630

28. Harrison RK (2010) Adela Ben-Yakar, "Role of near-field enhancement in plasmonic laser nanoablation using gold nanorods on a silicon substrate". Opt. Express 18:22556-22571

29. Nedyalkov NN, Takada H, Obara M (2006) Nanostructuring of silicon surface by femtosecond laser pulse mediated with enhanced near-field of gold nanoparticles. Appl. Phys. A 85:163-168 
30. Plech A, Kotaidis V, Lorenc M, Boneberg J (2006) Femtosecond laser near-field ablation from gold nanoparticles. Nature Phys. 2:44

31. Boutopoulos C, Dagallier A, Sansone M, Blanchard-Dionne A, Lecavalier-Hurtubise É, Boulais É, Meunier M (2016) Photoninduced generation and spatial control of extreme pressure at the nanoscale with a gold bowtie nano-antenna platform. Nanoscale 8(39):17196-17203

32. Thomas R, Sreejith S, Joshi H, Pedireddy S, Stupary MC, Zhao Y, Boon SC (2016) Optically induced structural instability in gold-silica nanostructures: a case study. Phys. Chem. C 120(20):11230-11236

33. Zuev DA, Makarov SV, Mukhin IS, Milichko VA, Starikov SV, Morozov IA, Shishkin II, Krasnok AE, Belov PA (2016) Fabrication of hybrid nanostructures via nanoscale laser-induced reshaping for advanced light manipulation. Adv. Mat 28(16):3087-3093

34. Leiderer P, Bartels C, König-Birk J, Mosbacher M, Boneberg J (2004) Imaging optical near-fields of nanostructures. Appl. Phys. Lett. 85:5370
35. Baffou G, Girard C, Quidant R (2010) Mapping heat origin in plasmonic structures. Phys. Rev. Lett. 104:136805

36. Dombi P, Irvine SE, Rácz P, Lenner M, Kroó N, Farkas G, Mitrofanov A, Baltuška A, Fuji T, Krausz F, Elezzabi AY (2010) Observation of few-cycle, strong-field phenomena in surface plasmon fields. Opt. Express 18:24206

37. Rácz P, Irvine SE, Lenner M, Mitrofanov A, Baltuška A, Elezzabi AY (2011) P Dombi, "Strong-field plasmonic electron acceleration with few-cycle, phase-stabilized laser pulses". Appl. Phys. Lett. 98: 111116

38. Budai J, Pápa Z, Márton I, Wróbel P, Stefaniuk T, Márton Z, Rácz P, Dombi P (2018) Plasmon-plasmon coupling probed by ultrafast, strong-field photoemission with $<7$ Angström sensitivity. Nanoscale 10:16261-16267

Publisher's Note Springer Nature remains neutral with regard to jurisdictional claims in published maps and institutional affiliations. 